Críston Pereira de Souza

\title{
Políticas Eficientes para Revisitação de Páginas Web
}

Tese de Doutorado

Tese apresentada ao Programa de Pós-graduação em Informática do Departamento de Informática da PUC-Rio como requisito parcial para obtenção do título de Doutor em Informática

Orientador: Prof. Eduardo Sany Laber 


\title{
Críston Pereira de Souza
}

\section{Políticas Eficientes para Revisitação de Páginas Web}

Tese apresentada ao Programa de Pós-graduação em Informática do Departamento de Informática do Centro Técnico Científico da PUC-Rio como requisito parcial para obtenção do título de Doutor em Informática. Aprovada pela Comissão Examinadora abaixo assinada.

\author{
Prof. Eduardo Sany Laber \\ Orientador \\ Departamento de Informática - PUC-Rio \\ Prof. Artur Alves Pessoa \\ Departamento de Engenharia de Produção - UFF \\ Prof. Marcus Felipe Fontoura \\ Yahoo! Research
}

Prof. Marcus Vinicius Soledade Poggi de Aragão

Departamento de Informática — PUC-Rio

Prof. Raúl Pierre Rentería Departamento de Informática — PUC-Rio

Prof. José Eugenio Leal

Coordenador Setorial do Centro Técnico Científico - PUC-Rio 
Todos os direitos reservados. É proibida a reprodução total ou parcial do trabalho sem autorização da universidade, do autor e do orientador.

\section{Críston Pereira de Souza}

Bacharel em Ciência da Computação pela Universidade Federal da Bahia e Mestre em Informática pela Pontifícia Universidade Católica do Rio de Janeiro. Tem experiência profissional como analista de sistemas, e atuou em projetos de pesquisa nas áreas de Otimização Combinatória, Aprendizado de Máquina e Sistemas Distribuídos.

Ficha Catalográfica

Souza, Críston Pereira de

Políticas eficientes para revisitação de páginas Web / Críston Pereira de Souza; orientador: Eduardo Sany Laber. Rio de Janeiro: PUC, Departamento de Informática, 2010.

v., 84 f: il. ; $29,7 \mathrm{~cm}$

1. Tese (doutorado) - Pontifícia Universidade Católica do Rio de Janeiro, Departamento de Informática.

Inclui referências bibliográficas.

1. Informática - Teses. 2. Máquinas de Busca. 3. Coletores Web. 4. Internet. 5. Algoritmos Aproximativos. 6. Simulação. I. Laber, Eduardo Sany. II. Pontifícia Universidade Católica do Rio de Janeiro. Departamento de Informática. III. Título. 


\section{Agradecimentos}

Ao meu orientador Professor Eduardo Laber pelo tempo dedicado durante todos estes anos, ajudando com muitas idéias e sua forma objetiva de trabalhar.

Ao CNPq, à FAPERJ e à PUC-Rio, pelos auxílios concedidos que permitiram realizar este trabalho.

À minha mãe Silene, que não mediu esforços para a educação dos filhos.

À Andréia pelo companheirismo e tantas ajudas importantes, sem os quais tudo seria mais difícil.

Aos colegas do LEARN/PUC-Rio pelos momentos de descontração, em especial os alunos Eduardo e Caio que colaboraram com esta pesquisa.

Aos funcionários do departamento de informática pela orientação e auxílio nas questões relacionadas ao curso.

Aos Professores Macêdo, Aline, Flávio e George do LaSiD/UFBA, por despertar em seus alunos o interesse pela pesquisa científica. 


\section{Resumo}

Souza, Críston Pereira de; Laber, Eduardo Sany. Políticas Eficientes para Revisitação de Páginas Web. Rio de Janeiro, 2010. 84p. Tese de Doutorado — Departamento de Informática, Pontifícia Universidade Católica do Rio de Janeiro.

Uma máquina de busca precisa constantemente revisitar páginas $W e b$ para manter seu repositório local atualizado. Uma política de revisitação deve ser empregada para construir um escalonamento de revisitações que mantenha o repositório o mais atualizado possível utilizando os recursos disponíveis. Para evitar sobrecarga de servidores Web, a política de revisitação deve respeitar um tempo mínimo entre requisições consecutivas a um mesmo servidor. Esta regra é chamada restrição de politeness. Devido ao porte do problema, consideramos que uma política de revisitação é eficiente se o tempo médio para escalonar uma revisitação é sublinear no número de páginas do repositório. Neste sentido, quando a restrição de politeness é considerada, não conhecemos política eficiente com garantia teórica de qualidade. Nesta pesquisa investigamos três políticas eficientes que respeitam a restrição de politeness, chamadas MERGE, RANDOM e DELAYED. Fornecemos fatores de aproximação para o nível de atualização do repositório quando empregamos as política MERGE ou RANDOM. Demonstramos que 0,77 é um limite inferior para este fator de aproximação quando empregamos a política RANDOM, e apresentamos uma conjectura de que 0,927 é um limite inferior para este fator de aproximação quando empregamos a política MERGE. As políticas também são avaliadas através da simulação da execução destas políticas para manter o nível de atualização de um repositório contendo 14,5 milhões de páginas Web. Um repositório contendo artigos da Wikipedia também é utilizado nos experimentos, onde podemos observar que a política MERGE apresenta melhores resultados que uma estratégia gulosa natural para este repositório. A principal conclusão desta pesquisa é que existem políticas simples e eficientes para o problema de revisitação de páginas $W e b$, que perdem pouco em termos do nível de atualização do repositório mesmo quando consideramos a restrição de politeness.

\section{Palavras-chave}

Máquinas de Busca; Coletores Web; Internet; Algoritmos Aproximativos; Simulação. 


\section{Abstract}

Souza, Críston Pereira de; Laber, Eduardo Sany (Advisor). Efficient Web Page Refresh Policies. Rio de Janeiro, 2010. 84p. DSc Thesis — Departamento de Informática, Pontifícia Universidade Católica do Rio de Janeiro.

A search engine needs to continuously revisit web pages in order to keep its local repository up-to-date. A page revisiting schedule must be defined to keep the repository up-to-date using the available resources. In order to avoid web server overload, the revisiting policy must respect a minimum amount of time between consecutive requests to the same server. This rule is called politeness constraint. Due to the large number of web pages, we consider that a revisiting policy is efficient when the mean time to schedule a revisit is sublinear on the number of pages in the repository. Therefore, when the politeness constraint is considered, there are no existing efficient policies with theoretical quality guarantees. We investigate three efficient policies that respect the politeness constraint, called MERGE, RANDOM and DELAYED. We provide approximation factors for the repository's up-to-date level for the MERGE and RANDOM policies. Based on these approximation factors, we devise a 0.77 lower bound for the approximation factor provided by the RANDOM policy and we present a conjecture that 0.927 is a lower bound for the approximation factor provided by the MERGE policy. We evaluate these policies through simulation experiments which try to keep a repository with 14.5 million web pages up-to-date. Additional experiments based on a repository with Wikipedia's articles concluded that the MERGE policy provides better results than a natural greedy strategy. The main conclusion of this research is that there are simple and efficient policies that can be applied to this problem, even when the politeness constraint must be respected, resulting in a small loss of repository's up-to-date level.

\section{Keywords}

Search Engines; Web Crawlers; Internet; Approximation Algorithms; Simulation. 


\section{Sumário}

1 Introdução 11

$\begin{array}{lll}1.1 & \text { Objetivo e Justificativa } & 16\end{array}$

$\begin{array}{lll}1.2 & \text { Metodologia } & 17\end{array}$

$\begin{array}{lll}1.3 & \text { Contribuições } & 17\end{array}$

1.4 Organização da Tese 20

2 Fundamentação Teórica e Trabalhos Relacionados 21

2.1 Medidas para o Nível de Atualização do Repositório 21

2.2 Restrição de Politeness 27

2.3 Políticas de Revisitação 28

2.4 Modelo Adotado nesta Tese 34

3 Caracterização do Repositório Experimental 35

3.1 Construção e Monitoramento 36

3.2 Número de Páginas por Servidor 38

3.3 Modificações segundo um Processo de Poisson 39

3.4 Distribuição das Taxas de Modificação 40

4 Política de Tempo Igualmente Espaçada por Página 41

4.1 Política de Tempo Igualmente Espaçada por Página 42

4.2 Política de Tempo DELAYED 44

4.3 Limites Superiores para o Freshness do Repositório 45

4.4 Alocação de Recursos considerando a Restrição de Politeness 47

4.5 Resultados Experimentais 50

5 Política de Tempo Igualmente Espaçada por Servidor 52

5.1 Política de Tempo Igualmente Espaçada por Servidor 54

5.2 Política de Seleção de Páginas RANDOM 55

5.3 Política de Seleção de Páginas MERGE 58

5.4 Uso Efetivo do Canal de Comunicação 63

5.5 Resultados Experimentais 66

6 Experimentos com um Repositório de Artigos da Wikipedia 68

6.1 Repositório WIKIPEDIA 69

6.2 Políticas de Revisitação 72

6.3 Experimentos $\quad 75$

7 Conclusões $\quad 78$

$\begin{array}{lll}7.1 & \text { Trabalhos Futuros } & 79\end{array}$

$\begin{array}{lr}\text { Referências Bibliográficas } & 81\end{array}$ 


\section{Lista de figuras}

1.1 Principais elementos do problema de revisitação de páginas Web. 12

2.1 Freshness e age de uma página. O freshness vale 1 quando a página está atualizada, ou 0 caso contrário. $O$ age vale 0 quando a página está atualizada, ou vale o tempo deste a última modificação quando está desatualizada.

3.1 Concentração das páginas nos servidores com mais páginas (repositório WEBBASE).

3.2 Concentração de páginas por servidor. Para cada quantidade $x$ de páginas por servidor, o gráfico fornece a proporção das páginas hospedadas em servidores com até $x$ páginas (repositório WEBBASE).

3.3 Histograma das durações dos intervalos entre modificações das páginas que se modificam em média 1 vez a cada 10 dias (repositório WEBBASE).

3.4 Distribuição das taxas de modificação das páginas - lei de potências (repositório WEBBASE).

4.1 Duas páginas de um mesmo servidor com atualizações igualmente espaçadas. $P$ é o tempo mínimo permitido entre requisições a um servidor. Pode ocorrer violação da restrição de politeness.

4.2 Política de tempo DELAYED: a $(i+1)$-ésima requisição ao servidor é escalonada para o instante $t_{i+1}$ e viola a restrição de politeness, sendo portanto atrasada para o instante $t_{i}+P$, onde $P$ é o tempo mínimo permitido entre requisições a um servidor.

4.3 Pseudo-código da política de tempo DELAYED.

4.5 Freshness do repositório WEBBASE fornecido pela política DELAYED durante 4 anos de operação do crawler, para a frequência total $C$ de revisitação igual a $1 \%, 10 \%$ e $90 \%$ da frequência máxima permitida pela restrição de politeness.

5.1 Pseudo-código da política de tempo igualmente espaçada por servidor. 54

5.2 Fator de aproximação para o freshness de uma página $i$ modificada com taxa $\lambda_{i}$, e revisitada com frequência $f_{i}$ pela política RANDOM. 56

5.3 Exemplo de aplicação da política de seleção de páginas MERGE. Duas páginas de um mesmo servidor são revisitadas com frequências $f_{1}$ e $f_{2}$. A frequência de requisições ao servidor vale $f=f_{1}+f_{2}$. Os deslocamentos $d_{1}, d_{2}$ e $d$ são uniformemente distribuídos nos intervalos $\left[0, f_{1}^{-1}\right),\left[0, f_{2}^{-1}\right)$ e $\left[0, f^{-1}\right)$, respectivamente.

5.4 Pseudo-código da política de seleção de páginas MERGE.

5.5 Revisitações igualmente espaçadas que ocorrem em um intervalo $I_{t}$ com duração $t$. 
5.6 Limite inferior fornecido pela Equação (5-9) para o fator de aproximação do freshness do repositório quando empregamos a política de seleção de páginas MERGE.

5.7 Limite superior para a probabilidade de ocorrer pelo menos $x$ requisições acima da quantidade esperada em um intervalo de tempo arbitrário. As requisições são igualmente espaçadas para cada um dos $n$ elementos.

5.8 Freshness do repositório WEBBASE fornecido pela política DELAYED durante 6 anos de operação do crawler, para a frequência total $C$ de revisitação igual a 1\%, 10\% e $90 \%$ da frequência máxima permitida pela restrição de politeness.

6.1 Distribuição acumulada empírica do tempo entre modificações consecutivas (em minutos) de 10 artigos escolhido aleatoriamente dentre os artigos do repositório WIKIPEDIA. Os gráficos mostram também as distribuições Pareto, Gama e Weibull ajustadas com estimadores de máxima verossimilhança.

6.2 Quando um artigo $i$ é avaliado no instante $t$, conhecemos o instante $u_{i}(t)$ da última revisitação deste artigo, e o instante $b_{i}(t)$ da sua última modificação antes de $u_{i}(t)$. $\mathrm{O}$ instante $A_{i}(t)$ da primeira modificação depois de $t$ é uma variável aleatória.

6.3 Freshness do repositório WIKIPEDIA após a execução das políticas MERGE, MERGE2, RANDOM e GREEDY, variando a frequência de requisições ao servidor. 
As far as the laws of mathematics refer to reality, they are not certain; and as far as they are certain, they do not refer to reality.

Albert Einstein, Sidelights on Relativity. 